\title{
Perfil clínico y epidemiológico de pacientes con hemofilia registrados en la Liga de Hemofílicos de Antioquia (Colombia).
}

\author{
Lina María Martínez-Sanchez, ${ }^{1}$ linam.martinez@upb.edu.co, Cardona-Velez Jonathan ${ }^{2}$, Ramirez-Pulgarin Sergio² ${ }^{2}$ Rodriguez-Gázquez
} María de los Ángeles ${ }^{1}$

Docente, Facultad de Medicina, Escuela de Ciencias de la Salud, Facultad de Medicina, Universidad Pontificia Bolivariana, Sede Central Medellín. Estudiante, Facultad de Medicina, Escuela de Ciencias de la Salud, Facultad de Medicina, Universidad Pontificia Bolivariana, Sede Central Medellín.

Fecha de recepción: agosto 28 de 2015

Fecha de correcciones: octubre 9 de 2015

Fecha de aceptación: julio 30 de 2016

Fecha de publicación: enero 31 de 2017

\begin{abstract}
Resumen
Introducción: La hemofilia es una enfermedad hemorrágica hereditaria caracterizada por la ausencia o deficiencia de factores VIII y IX de la coagulación. Objetivo. Determinar el perfil clínico y epidemiológico de los pacientes con hemofilia registrados en la liga de hemofílicos de Antioquia (LIHEA) durante 2005-2011. Metodología. Estudio descriptivo transversal, la población fueron pacientes con hemofilia de la LIHEA que cumplieron con criterios de selección. Se diseñó un instrumento de recolección de información y se aplicó el instrumento Modelo de actitud en pacientes con hemofilia (MAPACHE). Las variables cuantitativas se analizaron con medidas de tendencia central y dispersión, a las variables cualitativas se les estimó frecuencias absolutas, proporciones y razones. Resultados. Se incluyeron 36 hombres con hemofilia, con una mediana de 22 años de edad. En cuanto a los antecedentes personales los más frecuentes fueron HTA $(8.3 \%)$ y tromboflebitis (5.6\%). El sedentarismo fue el factor de riesgo de estilo de vida más común $(8.3 \%)$. Las manifestaciones clínicas más frecuentes fueron hemartrosis (94.4\%), hematomas musculares $(80.6 \%)$ y equimosis $(77.8 \%)$. En la mitad de los pacientes la enfermedad generó algún tipo de discapacidad, siendo la más frecuente la física $(44.4 \%)$. El tratamiento farmacológico más frecuente fue profilaxis con factor VIII con un $88.9 \%$, acompañado de la natación como principal tratamiento no farmacológico con un $77.8 \%$. En cuanto al MAPACHE el 30.6\% manifestó una actitud negativa hacia la enfermedad, un $5.6 \%$ fue neutra y para el $63.9 \%$ restante fue positiva. Conclusión. La hemofilia tuvo grandes repercusiones físicas y emocionales para los pacientes estudiados que la padecen.

Palabras clave: Hemofilia A, Hemofilia B, Hemorragia, Factores de Coagulación Sanguínea

Copyright (C) Facultad de Ciencias de la Salud de la Universidad Tecnológica de Pereira. 1995-2017. Todos los derechos reservados *.
\end{abstract}

Clinical and epidemiological profile of patients with hemophilia in Liga de Hemofílicos de Antioquia (Colombia).

\section{Abstract}

Introduction: Hemophilia is a hereditary hemorrhagic disorder characterized by the absence or deficiency of factors VIII and IX of the coagulation. Objective. To determine the clinical and epidemiological profile of patients with hemophilia recorded in the Liga de Hemofílicos de Antioquia (LIHEA) during 2005-2011. Methodology. Descriptive transversal study, population were patients with hemophilia from LIHEA which met selection criteria. We designed a data collection instrument and applied the attitude instrument in patients with hemophilia (MAPACHE). The quantitative variables were analyzed using measures of central tendency and dispersion, the qualitative variables were estimated absolute frequencies, proportions and reasons. This research had the respective ethical guarantee. Results. We included 36 men with hemophilia, with a median of 22 years of age. In terms of the personal history, the most frequent were hypertension (8.3\%) and thrombophlebitis (5.6\%). Sedentariness was the most common lifestyle risk factor $(8.3 \%)$. The most frequent clinical manifestations were hemarthrosis (94.4\%), muscle hematomas (80.6\%) and ecchymosis (77.8\%). In half of the patients, disease generated some kind of disability, being the most common the physics (44.4\%). The most common drug treatment was prophylactic factor VIII with $88.9 \%$, accompanied by swimming as the main non-pharmacological treatment with $77.8 \%$. Respect MAPACHE, the $30.6 \%$ expressed a negative attitude towards the disease, a $5.6 \%$ was neutral and the $63.9 \%$ was positive. Conclusion. Hemophilia is still a disease of great physical and emotional implications for studied patients who suffer it.

Keywords: Hemophilia A, Hemophilia B, Hemorrhage, Blood Coagulation Factors

Copyright $\odot$ Facultad de Ciencias de la Salud de la Universidad Tecnológica de Pereira. 1995-2017. All rights reserved ${ }^{\circ}$.

\section{Introducción}

La hemofilia es una enfermedad hemorrágica hereditaria que se caracteriza por la ausencia o deficiencia de los factores VIII y IX de la coagulación; hemofilia A y B respectivamente [1]. Según el World Federation of Hemophilia Global Survey de 2010 (WFH) existían en el mundo 162.781 casos de hemofilia, de estos 1915 casos reportados en Colombia, los cuales para su población representaba una prevalencia de 4.3 por 100.000 hombres, mucho mayor que la calculada para el mundo (2.6 por 100.000 hombres) [2].

A pesar de los avances médicos y las terapias de reemplazo con factores de coagulación, muchas de estas personas aún sufren los efectos crónicos de esta enfermedad; que afectan sustancialmente la calidad de vida [3-5]. En Colombia, según la WFH, se reportaron 1446 personas con Hemofilia tipo A, 303 tipo B y 145 personas fueron reportadas con hemofilia de origen desconocido [2]. Esta investigación tiene como objetivo determinar el perfil clínico y epidemiológico de los pacientes con hemofilia registrados en la liga de hemofílicos de Antioquia durante el periodo 2005-2011.

\section{Materiales y métodos}

Se realizó un estudio descriptivo, retrospectivo transversal en el cual la población de estudio estuvo conformada por aquellos pacientes diagnosticados con hemofilia A y B registrados en la Liga de hemofílicos de Antioquia (LIHEA) durante el periodo 2005-2011 y que cumplieron con los criterios de selección. Se diseñó un instrumento de recolección donde se registraron las variables clínicas y sociodemográficas del estudio, adicionalmente se aplicó el instrumento Modelo de actitud en pacientes con hemofilia (MAPACHE) de Carruyo y col. [6], con el fin de 
determinar la actitud. Este instrumento consta de catorce preguntas que exploran el componente cognitivo (fuerza de las creencias) y afectivo (contenido de las creencias), ambos con cinco opciones de respuesta; para el cognitivo: muy verdadero, verdadero, ni verdadero ni falso, falso y muy falso, y para el afectivo: muy bueno, bueno, ni malo ni bueno, malo y muy malo, los que se tradujeron a puntajes de $+2,+1,0,-1$ y -2 . Para el cálculo de la actitud se multiplicó el puntaje en cada una de las afirmaciones del componente cognitivo por su homóloga en el componente afectivo, por lo tanto el resultado de esta multiplicación estaría entre +4 y -4 . La actitud de cada individuo sería la sumatoria total del valor de las 14 afirmaciones y el signo establecería si la actitud es positiva o negativa. Se consideró actitud neutra si su valor numérico era igual a cero. Las variables cuantitativas se analizaron con las siguientes medidas descriptivas: se calcularon medidas de tendencia central y dispersión, según su distribución y a las variables cualitativas: se les estimó frecuencias absolutas, proporciones y razones. Se diseñó una base de datos en el programa SPSS versión 17, paquete con el cual se hizo el análisis de la información. En este estudio participaron menores de edad, los cuales siempre estuvieron acompañados de su acudiente. Esta investigación conto con el aval del Comité de Ética de la Escuela de Ciencias de la Salud de la Universidad Pontificia Bolivariana.

\section{Resultados}

En este estudio se incluyeron 36 hombres con hemofilia, la mediana de edad fue de 22 años (RIQ: 13.2-29, mínimo=6, Máxima=55), casi el $40 \%$ tenía menos de 19 años. Los pacientes estaban afiliados a la LIHEA con un RIQ: 5-25 años, mínimo=2, Máximo=29. Las características sociodemográficas de los pacientes pueden ser revisadas en la Tabla 1, apreciándose que en cuanto a la ocupación predominaron los estudiantes (47.2\%) y los estratos bajos (86.1\%). En relación a los antecedentes personales los más frecuentes fueron la hipertensión arterial (8.3\%) y la tromboflebitis (5.6\%). El sedentarismo fue el factor de riesgo de estilo de vida más común (8.3\%). Pasando al antecedentes familiar de hemofilia este estuvo presente en tres de cada cinco pacientes, siendo más frecuente en tíos y hermanos con un $27.8 \%$ y $13.9 \%$ respectivamente. De los pacientes con 18 y más años solo dos tenían hijos, de los que no tenían hijos actualmente: 12 piensan tenerlos y 7 no lo hará por conciencia del patrón de herencia de la enfermedad.

Tabla 1. Características sociodemográficas y antecedentes personales y familiares

\begin{tabular}{ccc}
\hline \multicolumn{1}{c}{ Característica } & $\%$ \\
\hline Edad & & \\
& $5-13$ & 25.0 \\
$14-18$ & 13.9 \\
$19-44$ & 50.0 \\
$45+$ & 11.1 \\
Ocupación & \\
Estudiante & 47.2 \\
Empleado & 33.3 \\
Trabajador independiente & 1.1 \\
Jubilado & 2.8 \\
Sin dato & 15.6 \\
1 & \\
Estrato socioeconómico & 11.1 \\
3 & 41.7 \\
4 & 33.3 \\
5 & 11.1
\end{tabular}

Antecedentes personales

Hipertensión arterial

Accidente cerebro vascular, angina y/o infarto

Dislipidemia

Tromboembolismo

5.6

Tromboflebitis $\quad 5.6$

Tabaquismo $\quad 2.8$

Sedentarismo $\quad 8.3$

Antecedentes familiares

Enfermedad cardiovascular $\quad 52.8$

Hemofilia * 61.1

Hermano 13.9

Tío $\quad 27.8$

Primo 11.1

Abuelo 11.1

En la Tabla 2 se pueden observar las características relacionadas con la hemofilia: nueve de cada diez tenían Hemofilia tipo A. En cuanto al momento de diagnóstico el $63.9 \%$ fue en el primer año de vida, el más precoz fue hecho a los 15 días de nacido y el más tardío a los 8 años de edad. Respecto a la clasificación según riesgo de sangrado para el $55.6 \%$ de los casos fue severa. Las manifestaciones clínicas con mayores proporciones fueron: hemartrosis (94.4\%) y hematomas musculares (80.6\%). Todos los pacientes en algún momento estuvieron hospitalizados por complicaciones relacionadas con la enfermedad, siendo la más frecuente la hemartrosis con un $80.6 \%$, las manifestaciones clínicas y sus complicaciones no fueron excluyentes. En la mitad de los pacientes la enfermedad generó algún tipo de discapacidad, siendo la más frecuente la física (44.4\%)

Tabla 2. Características relacionadas con la Hemofilia

\begin{tabular}{cc}
\hline \multicolumn{1}{c}{ Características } & $\%$ \\
\hline Tipo de hemofilia & \\
A & 91.7 \\
B & 8.3
\end{tabular}

Momento del diagnóstico

ler año 63.9

$2^{\circ}$ año $\quad 2.8$

3er año 16.7

$4^{\circ}$ año $\quad 5.6$

5 año $\quad 8.3$

8 año 2.8

Clasificación según riesgo de sangrado

Severo $\quad 55.6$

Leve $\quad 22.2$

Moderado $\quad 22.2$

Manifestaciones clínicas

Hemartrosis $\quad 94.4$

Hematomas musculares $\quad 80.6$

Equimosis $\quad 77.8$

Hemorragia digestiva $\quad 52.8$

$\begin{array}{ll}\text { Epistaxis } & 52.8\end{array}$

Hematuria $\quad 44.4$

Sangrado del SNC ${ }^{\dagger} \quad 16.7$

Otro $\quad 8.3$ 


\begin{tabular}{lc} 
Hospitalización por complicación & \\
Hemartrosis & 80.6 \\
Hematomas musculares & 58.3 \\
Hemorragia digestiva & 38.9 \\
Hematuria & 33.3 \\
Epistaxis & 19.4 \\
Sangrado del Sistema Nervioso Central & 16.7 \\
Equimosis & 2.8 \\
La enfermedad generó discapacidad & \\
Física & 44.4 \\
Cognitiva & 2.8 \\
Ambas & 2.8 \\
Sin discapacidad & 50.0 \\
\hline
\end{tabular}

El $88.9 \%$ de los pacientes recibían actualmente profilaxis con factor VIII y adicionalmente tratamiento no farmacológico, teniendo la natación, la fisioterapia y la psicoterapia las mayores proporciones $77.8 \%, 69.4 \%$ y $50.0 \%$, respectivamente. Uno de cada tres pacientes no continuo el tratamiento farmacológico, los principales motivos fueron las dificultades con la Empresa Prestadora de Salud - EPS (19.4\%), la indicación médica (5.6\%), los altos costos (5.6\%), y los efectos secundarios $(2.8 \%)$, ver Tabla 3.

Tabla 3. Características relacionadas con el tratamiento

Características $\%$

Tratamiento farmacológico en la actualidad

Profilaxis con factor VIII

88.9

Profilaxis con factor VII

Profilaxis con factor IX

Sin tratamiento actual

Tratamiento no farmacológico en la actualidad *

Natación

Fisioterapia

Psicología

50.0

Hidroterapia

47.2

Nutrición

36.1

Taller artesanal

Aeróbicos

Gimnasio

Tenis de mesa

2.8

En cuanto al test MAPACHE el $30.6 \%$ manifestó una actitud negativa hacia la enfermedad, un $5.6 \%$ fue neutra y para el $63.9 \%$ restante fue positiva. Los pacientes consideran bueno el comportamiento de su enfermedad en un $86.1 \%$, regular en un $8.3 \%$ y malo en el $5.6 \%$. El $80.6 \%$ piensa que puede llevar una vida completamente normal y un $61.1 \%$ en algún momento ha tenido dificultades para tener acceso a su tratamiento.

\section{Discusión}

La mediana de edad en el estudio de Recht y col. [7] fue de 16.2 años con un mínimo de 1.6 y un máximo de 60.9 , lo cual se asemeja al presente estudio donde la mediana fue de 22 años con un valor mínimo y máximo de 6 y 55 respectivamente. En cuanto a la distribución por edad, aproximadamente el $40 \%$ de los participantes de esta investigación tenían menos de 19 años, dato superior al 20\% reportado para Colombia [2].
Respecto a los datos sociodemográficos de ocupación en el estudio realizado en Brasil por Caio y col. [8] presentan que el 73\% de los pacientes hemofílicos tenían un empleo regular, el 23\% eran jubilados y un $4 \%$ sin dato, en este estudio los datos son inferiores con un $33.3 \%$ y un $2.8 \%$ para empleados y jubilados respectivamente.

La hemofilia se ha caracterizado por generar un estatus incapacitante en los pacientes, lo cual evidencian los diferentes estudios, en el estudio de Recht y col. [7] se reporta que muchos de los pacientes tenían alguna limitación para realizar actividades recreacionales, laborales y escolares, y solo el 28,2\% tenía un estatus funcional sin restricciones, mientras que en el presente estudio, en la mitad de los pacientes la enfermedad generó algún tipo de discapacidad, siendo la más frecuente la física con un $44.4 \%$ lo cual muestra valores equivalentes al estudio realizado por Carruyo y col. [6] con un 38\% de incapacidad para el trabajo y el deporte.

En el trabajo realizado por Recht y col. [7) reportaron que el $85 \%$ de los pacientes incluidos en el estudio fueron diagnosticados con hemofilia tipo A, Wang y col. [9] en su estudio un $84 \%$, algo similar se observa en el presente estudio donde el $90 \%$ de los pacientes tenían el mismo diagnóstico. A su vez el resultado obtenido en el presente estudio se asemeja al reporte entregado por WFH para Colombia con un $82 \%$ de personas con esta condición, demostrando ser de la hemofilia tipo A de mayor prevalencia.

En el trabajo de Evans y col. [10] se reportó que el $88.2 \%$ de los pacientes recibió tratamiento con facto VIII, 5.9\% con factor IX y el $5.9 \%$ restante no requirió ningún tratamiento, lo cual concuerda con los resultados obtenidos en este estudio con un total de $88.9 \%$ y $2.8 \%$ para los factores VIII y IX respectivamente y el 5.6\% recibió factor VII.

La hemofilia no solo afecta físicamente a los pacientes, sino que también tiene un alto componente psicosocial; este fue valorado con el test MAPACHE, según el estudio de Carruyo y col. [6] el $74.5 \%$ de los pacientes manifestaron una actitud positiva hacia su enfermedad, dato mayor al $63.9 \%$ encontrado en nuestro estudio.

La hemofilia es una patología que se destaca por ser una enfermedad de carácter hereditario, la cual compromete la población masculina afectando todos los grupos etarios, socioeconómicos y ocupacionales.

Esta condición es relevante para nuestro medio, no solo por las complicaciones que desarrolla, sino también por afectar a la población que se encuentran entre 19 a 44 años. En nuestro entorno es mayor la prevalencia de Hemofilia tipo A similar a la prevalencia mundial, de igual manera se evidencia claramente que es una patología heterogénea en donde las principales manifestaciones de estos pacientes son hemartrosis y hematomas musculares, aunque puede variar de una simple equimosis a un sangrado del sistema nervioso central.

Aunque el tratamiento en nuestro medio se brinda acorde a los estándares mundiales, sigue presentando problemas de tipo administrativo ya que el $25 \%$ de los pacientes abandona el tratamiento por problemas con la entidad prestadora de salud o problemas económicos personales. Aún con estas falencias al menos el 50\% de las personas no presentan ningún tipo de discapacidad. Es importante realizar estudios posteriores que permitan caracterizar mejor a la población y aportar mayor conocimiento de esta patología.

\section{Conflictos de interés}

Los autores declaran no tener conflictos de interés.

\section{Referencias}

1. Caicedo V, Sanabria R, Duarte R. Manejo perioperatorio del paciente hemofílico. Rev Mex Anest. 2009; 32(3):177-85.

2. World Federation of Hemophilia. Report on the annual global survey [Internet]. Montreal, Quebec: World Federation of Hemophilia; 2011 [acceso enero 23 2015]. Disponible en: http:// www1.wfh.org/publications/files/pdf-1574.pdf 
3. Pérez B, Ozelo M, Villaça P, Solano M, Jimenez C, Martínez M, et al. Diagnosis and treatment of congenital hemophilia with inhibitors a Latin American perspective. Medicina (B Aires). 2008 ; 68(3):227-42.

4. Chambost H. Assessing risk factors: prevention of inhibitors in haemophilia. Haemophilia. 2010;16 (Suppl 2):10-5.

5. Bermeo SM, Tamara SC, Fonseca DJ, Restrepo CM. Hemofilia: diagnóstico molecular y alternativas de tratamiento. Colomb Med. 2007; 38(3):308-5.

6. Carruyo C, Vizcaíno G, Carrizo E, Arteaga M, Sarmiento S, Vizcaíno J. Actitud de los individuos adultos con hemofilia hacia su enfermedad. Invest Clín. 2004;45(3): 257-6

7. Recht M, Neufeld EJ, Sharma VR, Solem CT, Pickard AS, Gut RZ, et al. Impact of acute bleeding on daily activities of patients with congenital hemophilia with inhibitors and their caregivers and families: observations from the Dosing Observational Study in Hemophilia (DOSE). Value Health. 2014; 17(6):744-8.

8. Caio VM, de Paiva e Silva RB, Magna LA, Ramalho AS. Genética comunitária e hemofilia em uma população brasileira. Cad Saude Publica. 2001;17(3):595-605

9. Wang J, Chan WC, Fu YC, Tong KM, Chang ST, Hwang WL, et al. Prevalence and risk factors of atherothrombotic events among 1054 hemophilia patients: A population-based analysis. Thromb Res. 2015. pii: S0049-3848(14)00696-3. [Epub ahead of print]

10. Evans M, Cottrell D, Shiach C. Emotional and behavioural problems and family functioning in children with haemophilia: a cross-sectional survey. Haemophilia. 2000;6(6):682-7. 